\title{
Future Time Perspective Scale (FTPS-P): Análise Rasch da versão Portuguesa Future Time Perspective Scale (FTPS-P): Portuguese version Rasch analysis
}

\author{
José Pacheco Miguel, Maria Paula Paixão, José Tomás da Silva, Teresa Sousa Machado \\ Universidade de Coimbra
}

\begin{abstract}
Resumo
O presente estudo analisa as propriedades psicométricas da Future Time Perspective Scale (FTPS) numa amostra de universitários portugueses, usando o modelo de Rasch. Os resultados indicam que os itens das subescalas ajustam a estruturas latentes unidimensionais, pressuposto requerido pelo modelo de Rasch. A escala de resposta apresentou funcionamento adequado, excepção feita à subescala de rapidez, permitindo que os parâmetros de pessoas e itens fossem estimados com elevada precisão. Análises DIF confirmaram a inexistência de diferenças nos resultados relativas ao género. São discutidas as limitações do estudo e as implicações psicométricas dos resultados para futuras investigações.

Palavras chave: perspectiva temporal, Rasch, rating scale model, DIF
\end{abstract}

\begin{abstract}
The present study analyzes the psychometric properties of the Future Time Perspective Scale (FTPS) in a sample of Portuguese university students with the Rasch model. The results indicate that subscale items are well fitted to latent unidimensional structures, as required by Rasch modeling. The response scale showed proper functioning with the exception of speed subscale; therefore, people and item parameters were estimated with high precision. DIF analyses confirmed no differences between genders in the results. Limitations of this study and the psychometric implications of its results are discussed, providing suggestions for future research.
\end{abstract}

Keywords: time perspective, Rasch, rating scale model, DIF

O estudo das percepções humanas acerca do futuro, sob a designação de Perspectiva Temporal de Futuro (PTF), tem atraído a atenção dos investigadores, na medida em que esta capacidade para considerar um tempo ainda não concretizado, esta disponibilidade de sacrifício no presente com o propósito de obter algo melhor no futuro, se assume como uma das principais estratégias responsáveis pelo nosso sucesso enquanto espécie. O grau em que o presente se liga ao passado e ao futuro, descreve o espaço de tempo que os sujeitos consideram quando tomam decisões acerca do seu presente e, neste contexto temporal, as necessidades do sujeito convertem-se em objectos (metas). Quanto mais o sujeito amplia a sua PTF, maior a quantidade de planos e metas que estabelece para atingir esses objectivos (Husman \& Lens, 1999).
Ainda assim, e apesar a evidência existente corroborar que a forma como os alunos universitários pensam acerca dos seus futuros tem impacto na sua persistência face ao estudo e no seu rendimento académico, a PTF só mais recentemente tem vindo a ser incorporada de forma mais ampla nas concepções de aprendizagem estratégica e autoregulada (Husman \& Shell, 2008). Uma das razões apontadas para que a psicologia educacional se revele deficitária quanto à avaliação das crenças dos estudantes relativamente ao tempo e às suas capacidades para o influenciarem ou gerirem, tem-se prendido com a falta de instrumentos adequados para medir a PTF em populações académicas.

Apesar da existência de instrumentos disponíveis para medir aspectos da PTF (Shell \& Husman, 2001), neles são reconhecidas fragilidades em termos de fidelidade e de validade de construto. A Future Time Perspective Scale (FTPS; Husman \& Shell, 2008) constitui, segundo as autoras, uma medida abrangente e psicometricamente robusta para a avaliar as dimensões subjacentes à PTF, de forma preferencial em alunos do ensino superior, razão pela qual foi adaptada à população Portuguesa (Cabral, 2011), sob supervisão da segunda autora.

Tendo em consideração que o primeiro estudo com a versão Portuguesa da escala (FTPS-P) foi realizado com alunos do ensino secundário e recorreu à teoria clássica dos testes (TCT), em termos metodológicos, optou-se por avaliar agora as suas propriedades psicométricas no âmbito da teoria de resposta ao item (TRI). De forma específica, optou-se pelo modelo de Rasch que, pelas suas propriedades métricas, usa apenas as respostas dos sujeitos enquanto estatísticas suficientes para estimar os parâmetros relativos à dificuldade dos itens e ao nível de atributo (PTF) detido pelos sujeitos, de forma simultânea e independente (Wright \& Mok, 2004).

\section{Método}

Foram realizadas análises de Rasch, com o software Winsteps 3.73.0 (Linacre, 2011). Especificamente, dado o formato politómico invariante de todos os itens da escala, as estimativas dos parâmetros relativos aos itens, aos sujeitos e às categorias de resposta foram calculadas com o Rating Scale Model (RSM, Wright \& Masters, 1982). O RSM é uma extensão do modelo de Rasch para itens politómicos e que, segundo Linacre (2002), tem a seguinte formalização matemática: 


$$
\log \left[P_{n i k} / P_{n i(k-1)}\right]=B_{n}-D_{i}-F_{k}
$$

$P_{\text {nik }}$ representa a probabilidade da pessoa $n$ responder na categoria $k$ do item $i, P_{n i(k-1)}$ é a probabilidade da pessoa $n$ responder na categoria $k-1$ do item $i, B_{n}$ é a medida da pessoa $n$ no traço avaliado, $D_{i}$ é a dificuldade do item $i$ e $F_{k}$ é a dificuldade do passo da categoria $k-1$ para a categoria $k$ (i.e., calibração do passo); esta calibração do passo $\left(F_{k}\right)$ é um limiar da escala de classificação definido como sendo a localização correspondente à equiprobabilidade de observação das categorias adjacentes $k-1$ e $k$.

A opção pelo RSM explica-se, para além das suas já referidas propriedades métricas que permitem que a comparação das pessoas seja independente dos itens usados para as medir e que a comparação dos itens seja invariante das pessoas utilizadas para os calibrar (objectividade específica), porque transforma os dados ordinais relativos às respostas dos sujeitos numa escala intervalar (Wright \& Mok, 2004). Em termos práticos, para além de apresentar estatísticas para o ajustamento da pessoa e do item, tem a vantagem de não necessitar de amostras grandes para estimar os seus parâmetros. Para além disso, permite ainda que se consiga determinar de forma empírica a qualidade discriminativa das categorias nas escalas de resposta de tipo Likert (Bond \& Fox, 2007).

A avaliação da FTPS-P com o RSM centrou-se nos aspectos da validade de conteúdo, da validade estrutural e da validade substantiva, propostos por Wolfe \& Smith (2007), com base no trabalho de Messick (1995).

\section{Participantes}

Participaram no estudo 254 alunos dos $1^{\circ}$ e $2^{\circ}$ anos de diferentes cursos da Universidade de Coimbra. As idades desta amostra não probabilística variaram entre um mínimo de 18 e um máximo de 25 anos, correspondendo a uma média de $20.67(D P=3.34)$. A distribuição é equilibrada quanto ao género, embora favoreça o sexo feminino $(N=134,52.8 \%)$. Não foi recolhida informação sobre a etnia por não ser uma variável relevante para os estudos realizados em Portugal (a maioria dos respondentes eram caucasianos europeus).

\section{Instrumentos}

O presente estudo integra um projecto de investigação alargado que incluiu uma bateria de instrumentos para avaliar várias medidas psicológicas (imprevisibilidade familiar, aspirações de carreira e satisfação com a vida), para além da PTF. Foi também informação demográfica (sexo, idade, sucesso escolar e habilitações académicas dos pais).

A Future Time Perspective Scale (FTPS; Husman \& Shell, 2008) é uma medida de auto-relato que visa avaliar aspectos relacionados com a perspectiva temporal global, através de 27 itens, repartidos por quatro subescalas, aos quais os sujeitos respondem numa Likert de cinco pontos de ancoragem ( 1 = Discordo fortemente, 2 = Discordo, 3 = Neutro, $4=$ Concordo e $5=$ Concordo fortemente) .

As autoras usaram quatro dimensões correspondentes aos construtos tradicionalmente considerados como mais estáveis do conjunto das crenças relativas à PTF. Apesar da sua estabilidade relativa, a evidência indica que estes aspectos, enraizados no contexto sociocultural, podem mudar (Bond \& Smith, 1996).

A instrumentalidade (I), medida por 12 itens $(\alpha=.82)$, oito dos quais com formulação negativa, constitui um aspecto cognitivo da PTF que consiste na capacidade de estabelecer ligações entre as actividade presentes e os objectivos futuros (Shell \& Husman, 2001); sujeitos com forte PTF possuem maior probabilidade de realização destas ligações. A valência (Va), medida por 7 itens ( $\alpha=$ .72), um em formulação negativa, é operacionalizada como a importância que os sujeitos atribuem às metas atingíveis no futuro e está associada ao comportamento adaptativo e à motivação positiva em contextos educativos pós ensino secundário; a valorização de objectivos futuros de longo prazo é indicadora de PTF forte (Husman \& Lens, 1999). A velocidade (Ve), medida com 3 itens ( $\alpha$ $=.72$ ), todos em formulação negativa, consiste na capacidade de antecipar e planear o futuro, medindo a velocidade com que os sujeitos sentem a passagem do tempo; centrada na falta de uma planificação plena do futuro, por falta de regulação externa para gerir os próximos acontecimentos, está associada a uma forte PTF cognitiva (Shell \& Husman, 2001). A extensão(E), medida por com 5 itens $(\alpha=.74), 3$ em formulação negativa, define-se pela distância temporal a que o sujeito consegue projectar os seus pensamentos; permitindo diferenciar desejos, sonhos e objectivos, esta subescala mede a percepção da proximidade temporal dos objectivos (Husman \& Shell, 2008).

\section{Procedimento}

O estudo foi realizado durante o primeiro semestre do ano lectivo 2014/2015, em contexto de sala de aula, com uma duração aproximada de 25 minutos. Assegurou-se o consentimento informado dos participantes, tendo sido garantida a confidencialidade e o direito de abandono a qualquer momento sem penalização.

\section{Resultados}

A TRI, e por conseguinte o modelo de Rasch, assenta, entre outros, no princípio da unidimensionalidade; os itens de uma escala medem um único atributo, ou traço latente, de modo a que o desempenho do sujeito apenas é influenciado por um factor dominante (Bond \& Fox, 2007; Miguel, 2013). Na medida em que a FTPS avalia quatro dimensões independentes (Husman \& Shell, 2008), a análise dos dados com o RSM foi realizada de modo independente para cada uma das subescalas.

Com o propósito de determinar a validade estrutural da escala, calculou-se a Análise de Componentes Principais (ACP) dos resíduos padronizados depois de controlar a dimensão Rasch, para averiguar se os itens das subescalas correspondem ao construto definido (Smith, 2004). Face à falta de consenso nos critérios para a presença de uma dimensão secundária (Chou \& Wang, 2010), os autores optaram por considerar um eigenvalue sem exceder $2.0 \mathrm{e}$ uma variância explicada pelo primeiro componente dos resíduos superior a $20 \%$ enquanto indicadores da unidimensionalidade das subescalas da FTPS-P (Linacre, 2011). 
A Tabela 1, relativa aos resultados da Análise de Componentes Principais dos Resíduos (ACPR) das subescalas da FTPS-P, permite constatar que todas elas cumprem o critério da unidimensionalidade, uma vez que os componentes principais das respectivas dimensões explicam mais de $20 \%$ da variância total e que os eigenvalues para o primeiro contraste, em cada uma das dimensões analisadas, são baixos, já que nenhum deles excede o limiar de aceitabilidade psicométrica (2.0). As medidas são consideradas boas, uma vez que os resíduos padronizados não têm informação sistemática adicional (Fisher, 2007).

Tabela 1.

ACP das subescalas I, Va, Ve e E da FTPS-P

\begin{tabular}{|c|c|c|c|c|c|}
\hline Sube & calas & I & $\mathrm{Va}$ & $\mathrm{Ve}$ & E \\
\hline \multirow{2}{*}{$\begin{array}{c}\text { Componente } \\
\text { Principal }\end{array}$} & eigenvalues & 26.0 & 4.0 & 3.2 & 2.7 \\
\hline & $\begin{array}{l}\text { Variância } \\
\text { explicada }\end{array}$ & $31.7 \%$ & $36.5 \%$ & $51.6 \%$ & $34.9 \%$ \\
\hline \multirow{2}{*}{$\begin{array}{l}\text { Primeiro } \\
\text { Contraste }\end{array}$} & eigenvalues & 2.0 & 1.4 & 1.2 & 1.9 \\
\hline & $\begin{array}{l}\text { Variância } \\
\text { explicada }\end{array}$ & $11.1 \%$ & $12.0 \%$ & $19.4 \%$ & $24.3 \%$ \\
\hline
\end{tabular}

As análises de Rasch proporcionam indicadores que permitem quantificar o ajustamento dos dados ao modelo, estimar os parâmetros do item e da pessoa e diagnosticar o funcionamento das categorias de resposta aos itens (Fox \& Jones, 1991). As Tabelas 2A, 2B, 2C e 2D apresentam as estatísticas dos itens para o ajustamento (infit e outfit), a localização $\left(D_{i}\right)$ e o erro padrão $(E P)$ que permitem avaliar a validade de conteúdo das subescalas da FTPS-P, bem como as respectivas média $(M)$ e desvio padrão $(D P)$, para além dos respectivos coeficientes de validade estrutural (CE) (Wolfe \& Smith, 2007).

Em termos de validade de conteúdo, as Tabelas $2 \mathrm{~A}$ a 2D permitem constatar que os valores médios de infit $\mathrm{e}$ outfit igualam ou quase coincidem com o valor esperado de 1.0 que indica um ajustamento perfeito dos itens; todos os itens da escala revelam valores individuais dentro do intervalo [.5 - 1.5] que Wright e Linacre (1994) estipulam como produtivo para a medida; indicia a inexistência de itens redundantes e a existência de homogeneidade entre os itens de cada uma das subescalas da FTPS-P. Este indicador da unidimensionallidade é confirmado por outros estimadores da modelação Rasch, com o fim de analisar o contributo dos itens na definição de um construto central para a estrutura interna da escala. De facto, as correlações ponto-medida $\left(r_{p m}\right)$, similares às correlações item-total da teoria clássica dos testes (TCT), com valores entre .43 e .63 , subescala I, entre .32 e .67 , subescala Va, entre .58 e .79 , subescala Ve e entre .43 e .62, subescala $E$, sugerem a inexistência de ruído não modelado ou de dependência nos dados (Linacre, 2011), permitindo concluir que cada um dos itens contribui para definir um mesmo construto comum (instrumentalidade, valência, velocidade e extensão, respectivamente).

Complementarmente, os valores de erro-padrão dos itens, variando entre .06 e .07 nas quatro subescalas, indicam que os itens mediram com elevada fiabilidade dentro de cada uma das subescalas. A TRI disponibiliza ainda, em termos de fiabilidade da medida, dois índices para se examinar a proporção de variância dos itens e dos sujeitos que não é explicada pelo erro-padrão da medida, a item separation reliability (precisão de separação dos itens) e a person separation reliability (precisão de separação dos sujeitos); esta última é um valor homólogo ao coeficiente alfa (Prieto \& Delgado, 2003).

Tabela 2A.

Propriedades psicométricas da subescala I da FTPS-P

\begin{tabular}{crrrrrr}
\hline & \multicolumn{2}{c}{ MNSQ } & & & & \\
\cline { 2 - 3 } Item & Infit & Outfit & \multicolumn{1}{c}{$D_{i}$} & $E P$ & $r_{p m}$ & CE \\
\hline 1 & 1.04 & 1.29 & .39 & .06 & .43 & -.03 \\
5 & .95 & .95 & -.26 & .06 & .43 & .60 \\
7 & .88 & .85 & -.13 & .06 & .57 & -.58 \\
8 & .76 & .79 & -.07 & .06 & .59 & -.57 \\
12 & .90 & .90 & -.27 & .06 & .46 & .20 \\
13 & 1.01 & 1.12 & .15 & .06 & .52 & -.46 \\
17 & 1.11 & 1.04 & .07 & .06 & .57 & .25 \\
19 & 1.23 & 1.25 & .09 & .06 & .43 & .67 \\
21 & 1.21 & 1.35 & -.16 & .06 & .45 & .15 \\
23 & .75 & .74 & -.05 & .06 & .63 & -.31 \\
25 & 1.18 & 1.20 & .19 & .06 & .54 & .06 \\
27 & .99 & .99 & .05 & .06 & .56 & -.17 \\
\hline M & 1.00 & .99 & .00 & .06 & & \\
DP & .16 & .20 & .19 & .00 & - & - \\
\hline
\end{tabular}

Tabela 2B.

Propriedades psicométricas da subescala Va da FTPS-P

\begin{tabular}{crrrrrr}
\hline & \multicolumn{2}{c}{ MNSQ } & & & & \\
\cline { 2 - 3 } Item & Infit & Outfit & \multicolumn{1}{c}{$D_{i}$} & $E P$ & $r_{p m}$ & CE \\
\hline 2 & .91 & .98 & .32 & .07 & .56 & -.49 \\
6 & 1.05 & 1.03 & -.41 & .07 & .53 & .66 \\
9 & .92 & .94 & -.16 & .07 & .58 & -.08 \\
14 & .92 & .93 & -.06 & .07 & .63 & .37 \\
15 & 1.51 & 1.78 & .66 & .07 & .32 & -.61 \\
18 & .72 & .70 & -.10 & .07 & .67 & -.06 \\
22 & .93 & .91 & -.25 & .07 & .64 & .47 \\
\hline M & 1.00 & 1.04 & .00 & .07 & & \\
DP & .23 & .32 & .34 & .00 & - & - \\
\hline
\end{tabular}


Tabela 2C.

Propriedades psicométricas da subescala Ve da FTPS-P

\begin{tabular}{|c|c|c|c|c|c|c|}
\hline \multirow[b]{2}{*}{ Item } & \multicolumn{2}{|c|}{ MNSQ } & \multirow[b]{2}{*}{$D_{i}$} & \multirow[b]{2}{*}{$E P$} & \multirow[b]{2}{*}{$r_{p m}$} & \multirow[b]{2}{*}{$\mathrm{CE}$} \\
\hline & Infit & Outfit & & & & \\
\hline 11 & 1.30 & 1.30 & .21 & .07 & .58 & 1.00 \\
\hline 16 & .89 & .85 & .30 & .07 & .73 & -.66 \\
\hline 26 & .79 & .75 & -.51 & .07 & .79 & -.60 \\
\hline $\mathrm{M}$ & .99 & .97 & .00 & .07 & & \\
\hline DP & .22 & .24 & .36 & .00 & & \\
\hline
\end{tabular}

Tabela 2D.

Propriedades psicométricas da subescala E da FTPS-P

\begin{tabular}{crrrrrr}
\hline & \multicolumn{2}{c}{ MNSQ } & & & & \\
\cline { 2 - 4 } Item & Infit & Outfit & \multicolumn{1}{l}{$D_{i}$} & $E P$ & $r_{p m}$ & CE \\
\hline 3 & .98 & 1.01 & -.35 & .06 & .55 & .39 \\
4 & 1.58 & 1.63 & -.04 & .06 & .43 & .77 \\
10 & .74 & .73 & -.16 & .06 & .66 & -.74 \\
20 & .76 & .73 & .31 & .07 & .62 & -.75 \\
24 & .98 & .95 & .24 & .07 & .57 & -.14 \\
\hline M & 1.01 & 1.01 & .00 & .06 & & \\
DP & .30 & .33 & .25 & .00 & - & - \\
\hline
\end{tabular}

Com valores que variam entre .54 , subescalas Ve e E, e .76, subescala I, a precisão de separação dos sujeitos é razoável (Fisher, 2007); a precisão de separação dos itens é boa, caso da subescala I (.89), ou mesmo muito boa, no caso das restantes subescalas da FTPS-P, com valores que variam entre .92 e .96 (Fisher, 2007). A precisão com que a escala mede e PTF é ajustada, embora que com uma maior fiabilidade ao nível dos itens do que das pessoas.

A validade substantiva refere-se ao diagnóstico do funcionamento empírico das categorias da escala de resposta, com o propósito de determinar se estas funcionam em conformidade com aquilo que era esperado pelo autor do instrumento quando desenvolveu os respectivos itens (Wolfe \& Smith, 2007). No contexto do RSM, Linacre (2002) propôs um conjunto de critérios para determinar a eficiência das categorias de resposta: (a) distribuição uniforme das frequências das respostas pelas diferentes categorias, com um mínimo de 10 observações em cada uma; (b) progressão monotónica da medida média observada $-B_{n}$ - e das calibrações dos passos $-F_{k}-$ ao longo das categorias de resposta; e (c) outfit MNSQ, mais sensível que o infit a respostas não esperadas, das categorias de resposta inferior a 2.0 .
Tabela 3A.

FTPS - P [1]: estatísticas das categorias de resposta

\begin{tabular}{ccrrrrrr}
\hline & \multicolumn{2}{c}{ Observada } & & \multicolumn{2}{c}{ MNSQ } & \\
\cline { 2 - 3 } Categoria & $f_{i}$ & $\%$ & $B_{n}$ & & Infit & Outfit & $F_{k}$ \\
\hline 1 & 261 & 9 & -.08 & 1.18 & 1.40 & - \\
2 & 399 & 13 & -.01 & .95 & .91 & -.52 \\
3 & 742 & 24 & .11 & .68 & .62 & -.52 \\
4 & 784 & 26 & .60 & .78 & .84 & .30 \\
5 & 861 & 28 & 1.06 & 1.06 & 1.08 & .74 \\
\hline
\end{tabular}

Tabela 3B.

FTPS-P [Va]: estatísticas das categorias de resposta

\begin{tabular}{|c|c|c|c|c|c|c|}
\hline \multirow[b]{2}{*}{ Categoria } & \multicolumn{2}{|c|}{ Observada } & \multirow[b]{2}{*}{$B_{n}$} & \multicolumn{2}{|c|}{ MNSQ } & \multirow[b]{2}{*}{$F_{k}$} \\
\hline & $f_{i}$ & $\%$ & & Infit & Outfit & \\
\hline 1 & 132 & 7 & -.56 & 1.18 & 1.25 & - \\
\hline 2 & 207 & 12 & -.20 & .90 & .87 & -.86 \\
\hline 3 & 620 & 35 & .19 & .82 & .79 & -1.06 \\
\hline 4 & 458 & 26 & .69 & .88 & .95 & .75 \\
\hline 5 & 361 & 20 & 1.12 & 1.07 & 1.17 & 1.18 \\
\hline
\end{tabular}

Tabela 3C.

FTPS_P [Ve]: estatísticas das categorias de resposta

\begin{tabular}{crrrrrrr}
\hline & \multicolumn{2}{c}{ Observada } & & \multicolumn{2}{c}{ MNSQ } & \\
\cline { 2 - 3 } Categoria & \multicolumn{1}{c}{$f_{i}$} & $\%$ & \multicolumn{1}{c}{$B_{n}$} & & Infit & Outfit & $F_{k}$ \\
\hline 1 & 64 & 8 & -1.38 & & 1.26 & 1.16 & - \\
2 & 208 & 27 & -.45 & 1.00 & .97 & -2.12 \\
3 & 202 & 27 & .16 & .96 & .92 & -.05 \\
4 & 144 & 19 & .86 & .98 & .94 & .90 \\
5 & 144 & 19 & 1.58 & .89 & .90 & 1.27 \\
\hline
\end{tabular}

Tabela 3D.

FTPS - P [E]: estatísticas das categorias de resposta

\begin{tabular}{ccccrrrr}
\hline & \multicolumn{2}{c}{ Observada } & & \multicolumn{2}{c}{ MNSQ } & \\
\cline { 2 - 3 } Categoria & $f_{i}$ & $\%$ & \multicolumn{1}{c}{$B_{n}$} & & Infit & Outfit & $F_{k}$ \\
\hline 1 & 352 & 28 & -1.13 & 1.05 & 1.07 & - \\
2 & 332 & 26 & -.63 & .91 & .97 & -.90 \\
3 & 299 & 24 & -.17 & .80 & .68 & -.30 \\
4 & 186 & 15 & .11 & .87 & .80 & .39 \\
5 & 101 & 8 & .19 & 1.23 & 1.34 & .81 \\
\hline
\end{tabular}

As Tabela 3A, 3B, 3C e 3D resumem as estatísticas necessárias para avaliar em que medida as 5 categorias de resposta da FTPS-P cumprem com estes critérios nas respectivas subescalas. A análise permite concluir que, genericamente, a estrutura da escala de resposta cumpre os critérios estipulados por Linacre (2002), uma vez que cada uma das categorias possui uma frequência observada de respostas superior a 10 e um valor de outfit inferior a 2.0; para além disso, a medida média observada 
e a calibração dos passos também aumentam de forma monotónica ao longo das 5 categorias de resposta, permitindo discriminar a amostra quanto ao atributo (PTF) que é medido. Ainda assim, o seu desempenho pode ser melhorado, porquanto nas subescalas I e $\mathrm{Va}$ as categorias 1 e 2 têm dificuldade em se diferenciarem.

O modelo de Rasch faz o escalonamento conjunto dos itens e dos sujeitos numa mesma dimensão (escala logit). Os parâmetros dos itens e dos sujeitos são expressos numa métrica comum, com as mesmas unidades (logits), numa escala de intervalos iguais, comparáveis entre si e localizados no mesmo contínuo (dimensão) (Embretson \& Reise, 2000). É desejável que as estimativas relativas ao atributo (pessoa) e à dificuldade (item) se sobreponham de forma substancial, para que o conjunto dos itens seja apropriado para a amostra. Sempre que a diferença entre as médias destas estimativas é inferior a 1 logit, significa que a informação contida nos itens permite discriminar as pessoas de forma precisa no construto que a escala se propõe medir (Bond \& Fox, 2007). No caso do presente estudo, o valor para essas diferenças varia entre cumpre o com critério nas subescalas I, Va, Ve e E $(.45, .53, .30$ e .57 , respectivamente).

Foi ainda analisado o funcionamento diferencial dos itens (DIF) para avaliar a validade dos resultados das subescalas da FTPS-P relativamente ao género. Calculou-se a diferença padronizada entre as localizações dos parâmetros masculino e feminino, depois de se terem ajustado as possíveis diferenças relativas ao sexo na distribuição da PTF, em termos de instrumentalidade, valência, velocidade e extensão; usou-se o procedimento de Bonferroni que corrigiu o nível de significância em função do número de comparações (.05/11) (Linacre, 2011). Seguindo este critério conservador, nenhum dos itens das subescalas I, Va, Ve e E revelou localização superior a .50, valor estipulado por Wright \& Douglas (1975) como ponto de corte para o contraste DIF, nem no sexo masculino, nem no sexo feminino. Conclui-se, pois, que os itens da FTPS-P têm funcionamento invariante quanto ao género.

\section{Discussão}

Globalmente, o estudo confirma que a FTPS-P é um instrumento válido na avaliação da perspectiva temporal de futuro dos alunos do ensino superior. Os resultados validam a unidimensionalidade das suas subescalas; a utilização do modelo de Rasch, permite que os níveis do construto das pessoas e de dificuldade com que os itens o medem sejam hierarquizados num contínuo escalar para as dimensões. Fá-lo com grau de precisão elevado que garante a adequação da escala de resposta, passível de ser refinada, e sem enviesamento quanto ao género.

A principal implicação deste estudo resulta, tal como os resultados comprovam, na demonstração da FTPS-P como medida multidimensional intervalar. $\mathrm{O}$ ajustamenno ao modelo de Rasch permite criar, para cada dimensão, uma escala linear (logits) para itens e pessoas que proporciona valores expressos nas mesmas unidades. Ao demonstrar que os itens e a amostra de calibração são independentes, permitindo comparar o nível de PTF do sujeito directamente com o nível de dificuldade do item usado para a medir, confirma a existência de estruturas simples, essencial para a medição invariante, base para modelos de medida úteis (Wright \& Masters, 1982).

O estudo apresenta algumas limitações que importa superar numa próxima investigação, designadamente no que diz respeito à amostra que, embora reproduzisse as características demográficas da população visada, não é representativa da realidade Portuguesa. Para que os resultados possam ser generalizáveis importa que no futuro se recorra a métodos de amostragem aleatória.

\section{Referências}

Bond, M.H. \& Smith, P.B. (1996). Cross-cultural social and organizational psychology. Annual Review of Psychology, 47, 205-235.

Bond, T.G., \& Fox, C.M. (2007). Applying the Rasch model. Fundamental measurement in the human sciences (2nd ed.). Mahwah, NJ: Lawrence Erlbaum.

Cabral, C. (2011). Perspectiva temporal de futuro e aspirações de vida: estudo das suas relações e do seu impacto na vitalidade, satisfação com a vida e exploração e compromisso com a carreira. Dissertação de Mestrado, Faculdade de Psicologia e de Ciências da Educação, Universidade de Coimbra, Coimbra, Portugal.

Chou, Y.-T., \& Wang, W.-C. (2010). Checking dimensionality in item response models with principal component analysis on standardized residual. Educational and Psychological Measurement, 70, 717-731. http:// dx.doi.org/10.1177/0013164410379322

Embretson, S.E. \& Reise, S.P. (2000). Item Response Theory for Psychologists. Mahwah, NJ: Lawrence Erlbaum Associates.

Fisher, W.P. Jr. (2007). Rating scale instrument quality criteria. Rasch Measurement Transactions, 21, p. 1095.

Fox, C.M., \& Jones, J.A. (1998). Uses of Rasch modeling in counseling psychology research. Journal of Counse- ling Psychology, 45, 30-45. http://dx.doi.org/10.1037// 0022-0167.45.1.30

Husman, J. \& Lens, W. (1999). The role of the future in student motivation. Educational Psychologist, 34(2), 113-125.

Husman, J. \& Shell, D.F. (2008). Beliefs and perceptions about the future: A measurement of future time perspective. Learning and Individual Differences, 18, 166-175.

Linacre, J.M. (2002). Optimizing rating scale category effectiveness. Journal of Applied Measurement, 3(1), 85-106.

Linacre, J.M. (2011). Winsteps Rasch measurement computer program, version 3.73.0. [Computer program] Chicago, Il: Winsteps.com.

Messick, S. (1995). Validity of psychological assessment: Validation of inferences from persons' responses and performances as scientific inquiry into score meaning. American Psychologist, 50, 741-749. http://dx.doi.org/ 10.1037//0003-066x.50.9.741

Miguel, J.P. (2013). TRI: representação e utilidade do modelo logístico de traço latente na psicometria actual. Dissertação de Doutoramento, Faculdade de 
Psicologia e de Ciências da Educação, Universidade de Coimbra, Coimbra, Portugal

Prieto, G. \& Delgado, A.R. (2003). Analisis de un test mediante el modelo de Rasch. Psicothema, 15(1), 94-100.

Shell, D.F. \& Husman, J. (2001). The multivariate dimensionality of personal control and future time perspective in achievement and studying. Contemporary Educational Psychology, 26, 481-506.

Smith Jr, E.V. (2004). Detecting and evaluating the impact of multidimensionality using item fit statistics and PCA of residuals. In: E.V. Smith Jr \& R.M. Smith (Eds.), Introduction to Rasch measurement (pp. 575-600). Maple Grove, MN: JAM Press.

Wright, B.D., \& Masters, G.N. (1982). Rating scale analysis. Chicago, Il: MESA Press.

Wolfe, E.W., \& Smith Jr, E.V. (2007). Instrument development tools and activities for measure validation using Rasch models: Part II - Validation activities. In E.V. Smith Jr \& R.M. Smith (Eds.), Rasch measurement: Advanced and specialized applications (pp. 243-290). Maple Grove, MN: JAM Press.

Wright, BD., \& Linacre, J.M. (1994). Reasonable mean-square fit values. Rasch Measurement Transactions, 8, p. 370.

Wright, B. D. \& Mok, M.M. (2004). An overview of the family of Rasch measurement models. In E.V. Smith Jr \& R.M. Smith (Eds.), Introduction to de Rasch Measurement (pp. 1-24). Maple Grove, MN: JAM Press.

\section{Agradecimentos}

Investigação realizada no âmbito do Laboratório de Psicometria da Faculdade de Psicologia da Universidade de Coimbra 